\title{
Eine expeditive Methode zum Nachweis von Galaktose und Milchzucker im Harn.
}

\author{
Von
}

Dr. Richard Bauer.

(Aus dem mediz.-chemischen Institut der Universität Wien.)

(Der Redaktion zugegangen am 5. Februar 1907.)

Durch meine Versuche über *alimentäre Galaktosurie und Laktosurie beim gesunden und kranken Menschen" ${ }^{1}$ ) bin ich vor die Aufgabe gestellt worden, Milchzucker und Galaktose im Harn nachzuweisen. Bei der Durchsicht der Literatur fand ich keine für meine Zwecke brauchbare Methode. Die Autoren haben sich meistens mit dem Nachweis der Wahrscheinlichkeit begnügt; dort, wo Milchzucker und Galaktose exakt nachgewiesen wurden, mußten überaus umständliche Verfahren verwendet werden.

So hat Hofmeister ${ }^{2}$ ) bei seinen Versuchen über alimentäre Galaktosurie beim Tiere die Galaktose nur durch die Gärungsprobe nachzuweisen versucht, desgleichen tat Voit, ${ }^{3}$ ) der beim Diabetiker aus der völligen Vergärung des Harnzuckers auf die Abwesenheit von Galaktose schloß. Bei den Versuchen über Laktosurie der Wöchnerinnen hat Hof meister ${ }^{4}$ ) den Zucker durch Bleifällung isoliert, den erhaltenen Sirup gereinigt und zur Krystallisation gebracht. Diese Krystalle wurden dann als reiner Milchzucker charakterisiert. Diese Methode ist zwar exakt, aber sie ist umständlich und gibt keine gute Ausbeute. Hofmeister ${ }^{4}$ ) konnte nur 50\% des Milchzuckers in reinem Zustande erhalten.

1) R. Bauer, Wiener mediz. Wochenschrift, 1906, Nr. 1.

2) Hofmeister, Archiv f. experim. Pathol. $u$. Therapie, 1885.

$\left.{ }^{3}\right)$ Voit, Zeitschrift f. Biologie, Bd. XXIX.

4) Hofmeister, Diese Zeitschrift, Bd. I. 
Da ich bei meinen Versuchen genötigt war, lange Zeit hindurch täglich viele Harnportionen einzeln auf Galaktose zu untersuchen, so habe ich mir eine Methode ausgearbeitet, die gestattet, die Galaktose im Harne exakt nachzuweisen, ohne daß es nötig ist, den Zucker vorher aus dem Harn zu isolieren. Ich habe diese Methode vielfach klinisch verwendet und über die klinische Seite meiner Versuche in der Wiener medizinischen Wochenschrift ${ }^{1}$ ) berichtet. Ich habe damals versprochen, an geeignetem Orte meine Methode ausführlich zu beschreiben und den Beweis für deren Brauchbarkeit zu erbringen, was hiermit geschehen soll. Bald darauf hat L. Langstein $\left.^{2}\right)$ Untersuchungen publiziert, bei denen er die Galaktose folgendermaßen nachgewiesen hat: Der Harn wurde mit salpetersaurem Quecksilberoxyd ausgefällt, aus dem Filtrat die Galaktose als Bleiverbindung isoliert, die Bleiverbindung mit Schwefelwasserstoff zersetzt und der erhaltene Galaktosesirup zu Schleimsäure oxydiert. Meine Methode ist einfacher; das Prinzip derselben ist, daß Milchzucker und galaktosehaltiger Harn direkt mit Salpetersäure oxydiert und die aus dem Zucker entstandene Schleimsäure abgeschieden wird.

Die Methode wird folgendermaßen ausgeführt:

$100 \mathrm{ccm}$ Harn werden mit $20 \mathrm{ccm}$ reiner konzentrierter Salpetersäure (spez. Gewicht 1,4) versetzt und in einem breiten, wenig tiefen Becherglase im siedenden Wasserbade eingedampft. Der Harn wird beim Zusatz der Salpetersäure gewöhnlich dunkel, wird aber beim Erwärmen bald wieder heller und bleibt so hell, bis er auf $40-50 \mathrm{ccm}$ eingedampft ist. In diesem Stadium beginnt die Flüssigkeit sich mehr braun $\mathrm{zu}$ färben, gerät in gelindes Schäumen, und es entweichen, insbesondere beim Schütteln, braune Dämpfe, zum Zeichen dafür, daß jetzt die Oxydation stattfindet. Diese Entwieklung von braunen Dämpfen dauert gewöhnlich solange an, bis der Harn auf ein Volumen von $20 \mathrm{ccm}$ eingedampft ist; dann wird die Flüssigkeit wieder

1) R. Bauer, Versuche über die Assimilation von Galaktose und Milchzucker beim Gesunden und Kranken, Wiener med. Wochenschrift, 1906, Nr. I.

2) Langstein u. Steinitz, Hofmeisters Beiträge, 1906, Bd. VII. 
heller, hört auf zu schäumen, und man sieht, daß sich in der gelben, früher klaren Flüssigkeit ein feiner weißer Niederschlag bildet, der rasch an Masse zunimmt. Man nimmt nun das Becherglas aus dem Wasserbade, überleert den Inhalt in ein kleineres Gläschen, wäscht zweimal mit etwas destilliertem Wasser nach. Der Niederschlag nimmt in der Kälte rasch $\mathrm{zu}$, weshalb man am besten die eingedampfte Flüssigkeit über Nacht an kühlem Orte stehen läßt. Nun wird mit Wasser verdünnt und der Niederschlag auf ein Filter gebracht. Nach wiederholtem Waschen mit destilliertem Wasser, eventuell Alkohol und Äther, wird der Niederschlag getrocknet und gewogen.

Der trockene Niederschlag zerfällt zu einem feinen weißen Pulver, das sich in kaltem Wasser beinahe gar nicht, in heißem Wasser nur schwer löst. In Alkohol, Äther, Chloroform, Benzol ist die Substanz unlöslich. Leicht löst sich dagegen das Pulver in verdünnten Lösungen von Natron- und Kalilauge, sowie in Lösungen von kohlensaurem Ammonium, in letzterem unter heftigem Aufschäumen (lebhafter Kohlendioxydentwicklung). Auf Säurezusatz fällt aus diesen Lösungen die Substanz als feiner Niederschlag langsam wieder aus. Das Pulver schmilzt in rohem Zustand bei $213-215^{\circ}$ unter Aufschäumen und Verkohlung. Manchmal liegt der Schmelzpunkt der rohen Substanz noch etwas tiefer. Krystallisiert man die Substanz aus heißem Wasser um, so erhält man ein glitzerndes, aus mikroskopisch kleinen Prismen bestehendes Pulver, das einen höheren Schmelzpunkt als die rohe Substanz besitzt. Dieser Schmelzpunkt ist jedoch nicht konstant, er wechselt zwischen 217 und $225^{\circ}$. Von zwei analysenreinen Präparaten schmolz eines bei $222^{\circ}$, eines bei $225^{\circ}$ bei gleichmäßig raschem Erhitzen. In zwei Versuchen wurde die Schleimsäure, wie oben geschildert, aus heißem Wasser umkrystallisiert, im Vakuum über Schwefelsäure getrocknet und zur Elementaranalyse verwendet.

Dieselbe ergab folgende Werte:

I. $0,2105 \mathrm{~g}$ Substanz gaben $0,2629 \mathrm{~g}$ Kohlendioxyd und 0,0912 $\mathrm{g}$ Wasser II. 0,1993 , > , 0,2508 , > 0,0858 , , 
Bei der Titration mit Lauge wurde gefunden: 0,2129 g Substanz verbrauchten $19,2 \mathrm{ccm} \mathrm{NaHO}$ $(1 \mathrm{ccm} \mathrm{NaHO}=1,0267 \mathrm{ccm} 1 / 10-N o r m a l-N a H O)$.

\begin{tabular}{|c|c|c|}
\hline & Berechnet & Gefunden \\
\hline $\begin{array}{l}\text { C . . . . . . . } \\
\text { H . . . . . . . } \\
\text { Äquivalentgewicht . }\end{array}$ & $\begin{array}{c}34,27 \% \\
4,8 \% \\
105\end{array}$ & $\begin{array}{c}\text { I. } 34,06 \% \text {; II. } 34,32 \% \\
\text { I. } 4,85 \% \text {; II. } 4,82 \% \\
108\end{array}$ \\
\hline
\end{tabular}

Die chemische Untersuchung lehrt also, daß der aus galaktosehaltigen Harnen durch Oxydation mit Salpetersäure erhaltene Niederschlag Schleimsäure ist.

Dagegen lehrten zahlreiche Kontrollversuche, daß man mit Harnen, die weder Galaktose noch Milchzucker enthalten, niemals einen derartigen Niederschlag erhält. Zahlreiche solche Harne von verschiedenem spezifischen Gewicht habe ich nach der beschriebenen Methode behandelt; in den meisten Fällen fiel überhaupt kein Niederschlag aus, in wenigen Fällen bildeten sich Krystalle von salpetersauren Salzen, die auf Zusatz von Wasser prompt in Lösung gingen.

Die oben geschilderte Methode wurde an mehr als hundert Harnportionen ausgeführt, die zum geringeren Teile künstlich mit Galaktose versetzt worden waren, zum größeren Teile von Patienten stammten, bei denen alimentäre Galaktosurie erzielt worten war. In den meisten Fällen wurde im Sinne obiger Ausführungen schon in der Wärme ein Schleimsäureniederschlag erhalten, nur in wenigen Fällen, wo kleine Mengen von Galaktose vorhanden waren, fiel die Schleimsäure erst nach einiger Zeit, in ganz vereinzelten Fällen. erst nach mehreren Stunden in der Kälte aus.

Die Ausbeute an Schleimsäure, die man bei dem beschriebenen Verfahren erhält, ist von mehreren Umständen abhängig, die aus der folgenden Tabelle ersichtlich sind.

Wie aus der Tabelle zu entnehmen ist, spielt vor allem die Menge der zugesetzten Salpetersäure eine Rolle. Man muß den Zusatz der Salpetersäure bemessen nach der Menge

Hoppe-Seyler's Zeitschrift f. physiol. Chemie. LI. 
Tabelle I.

\begin{tabular}{|c|c|c|c|c|c|c|c|}
\hline $\begin{array}{c}\text { Zum } \\
\text { Versuch } \\
\text { ver- } \\
\text { wendete } \\
\text { Harn- } \\
\text { menge } \\
\text { ccm }\end{array}$ & $\begin{array}{r}\text { Nach d } \\
\text { nissen } d \\
\text { und Po } \\
\text { Gal } \\
\text { in } \%\end{array}$ & $\begin{array}{l}\text { Ergeb- } \\
\text { 'itration } \\
\text { isation } \\
\text { se } \\
\text { in } \mathbf{g}\end{array}$ & $\begin{array}{r}\text { Ausb } \\
a \\
\text { Schlei } \\
\text { in } \%\end{array}$ & $\begin{array}{l}\text { ute } \\
\text { in } \mathrm{g}\end{array}$ & $\begin{array}{l}\text { Zuge- } \\
\text { setzte } \\
\text { Sal- } \\
\text { peter- } \\
\text { säure } \\
\text { in } \\
\mathrm{ccm}\end{array}$ & $\begin{array}{c}\text { Einge- } \\
\text { dampft } \\
\text { auf } \\
\mathrm{ccm}\end{array}$ & $\begin{array}{l}\text { Spezif. } \\
\text { Gewicht } \\
\text { des } \\
\text { Harnes }\end{array}$ \\
\hline 52 & 2,7 & 1,4 & 60 & 0,85 & 15 & 10 & - \\
\hline 100 & 1 & 1 & 60 & 0,6 & - & - & - \\
\hline 100 & 0,5 & 0,5 & 50 & 0,25 & - & - & - \\
\hline 100 & 1,3 & 1,3 & 65 & 0,85 & 10 & - & - \\
\hline 100 & 1,3 & 1,3 & 60 & 0,8 & 20 & - & - \\
\hline 150 & 0,5 & 0,75 & 27 & 0,2 & - & - & - \\
\hline 100 & 2 & 2 & 30 & 0,6 & 20 & - & - \\
\hline 76 & 0,33 & 0,25 & 40 & 0,1 & 6 & 5 & - \\
\hline 88 & 1,3 & 1,15 & 70 & 0,8 & 12 & 10 & - \\
\hline 180 & 0,37 & 0,7 & 35 & 0,25 & 20 & 15 & - \\
\hline 85 & 0,85 & 0,75 & 70 & 0,5 & 15 & 10 & 1004 \\
\hline 100 & 1,5 & 1,5 & 66 & 1 & 25 & 15 & - \\
\hline 100 & 2 & 2 & 70 & 1,4 & 25 & 20 & 1020 \\
\hline 50 & 2 & 1 & 70 & 0,7 & 12,5 & 10 & 1020 \\
\hline 100 & 2,7 & 2,7 & 80 & 2,15 & 30 & 20 & 1024 \\
\hline 100 & 1,15 & 1,15 & 70 & 0,8 & 20 & 15 & 1009 \\
\hline 50 & 2,2 & 1,1 & 60 & 0,65 & 12 & 8 & 1010 \\
\hline 60 & 1,1 & 0,66 & 75 & 0,5 & 15 & 5 & - \\
\hline 100 & 1,8 & 1,8 & 66 & 1,2 & 20 & 20 & 1013 \\
\hline 100 & 1,8 & 1,8 & 50 & 0,9 & 20 & $8-10$ & 1013 \\
\hline 50 & 5,52 & 2,76 & 60 & 1,66 & 25 & 20 & - \\
\hline 80 & 3,7 & 3 & 77 & 2,3 & - & - & - \\
\hline
\end{tabular}

der im Harn enthaltenen oxydierbaren Substanzen, für welche ja das spezifische Gewicht einen genügenden Anhaltspunkt liefert. Bei der alimentären Galaktosurie ist die Menge der im Harn gelösten Bestandteile gewöhnlich sehr gering, diese blassen Harne zeigen ein sehr niedriges spezifisches Gewicht (1003-1010, selten höher als 1020). Bedenkt man, daß hier 
ein großer Teil der gelösten Bestandteile Galaktose ist, so ergibt sich, daß nur sehr wenig andere oxydable Substanzen im Harn enthalten sein können.

Tabelle II.

Versuche mit Harnen, die Galaktose + Dextrose enthalten.

\begin{tabular}{c|c|c|c|c|c}
\hline $\begin{array}{c}\text { Zum Versuch } \\
\text { verwendete } \\
\text { Harnmenge } \\
\text { ccm }\end{array}$ & $\begin{array}{c}\text { Rechts- } \\
\text { drehung } \\
\text { in Prozenten } \\
\text { Trauben- } \\
\text { zucker }\end{array}$ & $\begin{array}{c}\text { Schleim- } \\
\text { säure } \\
\text { in g }\end{array}$ & $\begin{array}{c}\text { Der Schleim- } \\
\text { säure ent- } \\
\text { sprechen an } \\
\text { Galaktose ing } \\
\text { mindestens }\end{array}$ & $\begin{array}{c}\text { Zu- } \\
\text { gesetzte } \\
\text { Salpeter- } \\
\text { säure } \\
\text { ccm }\end{array}$ & $\begin{array}{c}\text { Ein- } \\
\text { gedampt } \\
\text { auf } \\
\text { ccm }\end{array}$ \\
\hline 100 & 8,9 & 2 & 2,5 & 35 & - \\
100 & 8,3 & 0,7 & 0,88 & 40 & 25 \\
100 & 4,8 & 0,5 & 0,62 & 35 & - \\
100 & 6,6 & 1,5 & 1,9 & 40 & -
\end{tabular}

In diesen, praktisch hauptsächlich in Betracht kommenden Fällen kommt man wohl immer zum Ziele, wenn man zur 0xydation dieser Harnbestandteile $10 \mathrm{ccm}$ und für jedes Prozent Zucker $4 \mathrm{ccm}$ Salpetersäure verwendet, so daß man gewöhnlich zu $100 \mathrm{ccm}$ Harn $20 \mathrm{ccm}$ Salpetersäure hinzuzusetzen hat. Ist das spezifische Gewicht höher als 1020, was gewöhnlich durch höheren Zuckergehalt bedingt ist, so muß man mehr Salpetersäure, etwa $25-35 \mathrm{ccm}$, zusetzen. Erfahrungsgemäß schadet ein mäßiger Überschuß an Salpetersäure nicht; wird hingegen zu wenig Salpetersäure hinzugesetzt, so wird selbstverständlich die Oxydation nicht zu dem gewünschten Ende geführt.

Es ist ferner zu überlegen, wie weit man den Harn eindampfen soll. Wie oben ausgeführt wurde, dampft man den Harn gewöhnlich auf ein Volumen ein, das dem Volumen der zugesetzten Salpetersäure entspricht, also bei Zusatz von $20 \mathrm{ccm}$ Salpetersäure auf $20 \mathrm{ccm}$. Maßgebend hierbei ist jedoch, daß die Oxydation, bezw. die Entwicklung von braunen Dämpfen, bereits stattgefunden hat. Wäre dies nicht der Fall gewesen, so muß man weiter eindampfen, wie dies auch aus der Tabelle ersichtlich ist.

Unter Einhaltung dieser Bedingungen gelingt es ausnahmslos, 
dort Schleimsäure zu erhalten, wo der Harn mindestens 10,0 Galaktose enthält. Enthält der Harn weniger Galaktose, so kommt man mit folgendem Kunstgriff zum Ziele: Man dampft nebeneinander mehrere Harnportionen unter Salpetersäurezusatz in der oben beschriebenen Weise auf je $20 \mathrm{ccm}$ ein, vereinigt die eingedampften Flüssigkeiten und dampft sie weiter ein, bis die braunen Dämpfe sich entwickeln. So gelingt es noch, $0,5 \%$, ja 0,25\% Galaktose als Schleimsäure nachzuweisen.

Die Ausbeute an Schleimsäure ist gut, aber nicht konstant. Haben ja schon die Versuche von Tollens ${ }^{1}$ ) gezeigt, daß reine Galaktose bei der Ox.ydation mit Salpetersäure nie die theoretisch berechnete Menge an Schleimsäure liefert, sondern selten mehr als 70\%, höchstens 80\% der angewandten Galaktosemenge. Allerdings erhält man aus dem Harn oft Mengen von Schleimsäure, welche $70-80 \%$ von der im Harn enthaltenen Galaktosemenge betragen (siehe Tabelle), aber oft auch nur $50-60 \%$.

Immerhin kann die Wägung der abgeschiedenen Schleimsäure einige wertvolle Aufschlüsse geben. Gesetzt den Fall, $100 \mathrm{ccm}$ Harn liefern bei der Oxydation mit Salpetersäure $0,8 \mathrm{~g}$ Schleimsäure und die mit dem Harn vorgenommene titrimetrische und polarimetrische Untersuchung ergibt Werte, welche einem Gehalt von 1\% Galaktose entsprechen, so kann daraus geschlossen weraen, daß in diesem Harn neben dem einen Prozent Galaktose kein anderer Zucker vorhanden war.

Für mich kamen vielfach Harne in Betracht, in welchen Galaktose neben Dextrose nachgewiesen werden sollte. ${ }^{2}$ ) Mein Verfahren hat sich auch in diesen Fällen anstandslos bewährt, was um so wertvoller ist, als hier alle anderen Methoden im Stiche lassen. Der Vergleich der bei der titrimetrischen und polarimetrischen Untersuchung gefundenen Werte lehrt höchstens, daß neben Dextrose noch ein anderer Zucker im Harn enthalten ist, gibt jedoch keinen Aufschluß über die Mengen der einzelnen Zuckerarten.

1) Handbuch der Kohlehydrate, I. B., S. 100, 318, Breslau 1898.

2) R. Bau er, Weitere Untersuchungen über alimentäre Galaktosurie, Wiener med. Wochenschrift 1906, Nr. 52. 
Das Gewicht der abgeschiedenen Schleimsäure hingegen läßt wenigstens einen approximativen Schluß auf die Menge der Galaktose $\mathrm{zu}$, so daß wenigstens ein Minimalwert für diese angegeben werden kann.

Die Methode kann naturgemäß, wie ich mich überdies durch Versuche überzeugen konnte, auch zum Nachweis von Milchzucker im Harn verwendet werden. Selbstverständlich kann sie allein nicht entscheiden, ob in dem Harn Galaktose oder Milchzucker enthalten war; doch kann auch hier, so wie in den früher skizzierten Fällen, die Menge der abgeschiedenen Schleimsäure verglichen mit den Resultaten der Untersuchung im Polarisationsapparat und durch Titration wertvolle Aufschlüsse geben über die.Natur und die Menge des vorhandenen Zuckers, während die beiden letztgenannten Untersuchungsmethoden allein auch hier im Stiche lassen, wie bei dem früher besprochenen Gemenge von Dextrose und Galaktose. Derartige Überlegungen haben mir oft bei meinen klinischen Arbeiten gute Dienste geleistet.

Praktisch kommt bei Wöchnerinnen die Frage in Betracht, ob ein mit dem Harn ausgeschiedener Zucker Dextrose oder nur Milchzucker ist. Ich beabsichtige weiter zu untersuchen, ob nicht auch hier meine Methode von Vorteil ist.

In obigen Ausführungen war von Gärversuchen nicht die Rede. Die Durchsicht der Literatur zeigte mir, daß in bezug auf das Gärungsvermögen der Galaktose sehr verschiedene Ansichten herrschen, weshalb ich danach strebte, mich von dieser Methode unabhängig zu machen. Dennoch habe ich der Vollständigkeit wegen in allen Fällen, wo ich die Galaktose als Schleimsäure nachgewiesen habe, auch den Gärversuch angestellt. Die durch Kochen sterilisierten Harne wurden nach dem Auskühlen mit frischer Preßhefe versetzt, in sterile Gärungsröhrchen gefüllt und in den Brutschrank gestellt.

Die Resultate waren folgende:

Galaktosehaltige Harne zeigen nach sechs Stunden keine oder nur geringe Gärung, während eine Kontrollprobe- mit 
Dextrose in derselben Zeit völlig vergärt. Dehnt man den Gärversuch auf längere Zeit aus, so beginnt auch der galaktosehaltige Harn langsam, aber deutlich $z u$ gären. In Harnen, die neben Galaktose auch Dextrose enthalten, schreitet die Gärung rascher vorwärts, es vergärt nicht nur die Dextrose, sondern auch die Galaktose kommt relativ rascher zur Vergärung. In rein wässerigen Lösungen gärt Galaktose weniger gut, es ist daher notwendig, bei Kontrollversuchen entweder der wässerigen Galaktoselösung einen Hefextrakt zuzusetzen oder die Galaktose in Harn aufzulösen. 